\title{
Erratum: Enskog kinetic theory for a model of a confined quasi-two-dimensional granular fluid [Phys. Rev. E 98, 052904 (2018)]
}

\author{
Vicente Garzó, Ricardo Brito $\odot$, and Rodrigo Soto
}

(Received 6 October 2020; published 3 November 2020)

DOI: 10.1103/PhysRevE.102.059901

Whereas working on the extension of the $\Delta$ model to granular mixtures, we have realized that the term $\frac{1}{2} \zeta^{(0)} \Delta^{*}\left(\partial \ln \zeta_{0}^{*} / \partial \Delta^{*}\right)$ must be also considered in the expression of the (reduced) kinetic thermal conductivity coefficient $\kappa_{\mathrm{k}}^{*}$ in the relevant state with stationary temperature (where $\zeta^{(0)}=0$ ). The inclusion of this term changes the expression of $\kappa_{\mathrm{k}}^{*}$ displayed in Table I of our paper. For a two-dimensional granular gas $(d=2), \kappa_{\mathrm{k}}^{*}$ is

$$
\kappa_{\mathrm{k}}^{*}=\frac{1+\frac{3}{8} \phi \chi(1+\alpha)^{2}(2 \alpha-1)-\frac{\Delta_{\mathrm{s}}^{*}}{\sqrt{2 \pi}} \phi \chi\left[\frac{7}{4}+2(1+\alpha)\left(1-\frac{3}{4} \sqrt{2 \pi} \Delta_{\mathrm{s}}^{*}\right)-3(1+\alpha)^{2}-\Delta_{\mathrm{s}}^{* 2}\right]}{2 v_{\kappa}^{*}+\Delta_{s}^{*}\left(\frac{\partial \zeta_{0}^{*}}{\partial \Delta^{*}}\right)_{s}},
$$

where

$$
\left(\frac{\partial \zeta_{0}^{*}}{\partial \Delta^{*}}\right)_{s}=-\frac{1}{2} \chi\left(\sqrt{2 \pi} \alpha+4 \Delta_{s}^{*}\right)
$$

Upon obtaining Eq. (2), we recall that the zeroth-order contribution to the (reduced) cooling rate $\zeta_{0}^{*}$ has been evaluated by neglecting non-Gaussian corrections to the zeroth-order distribution function $\left(a_{2, \mathrm{~s}}=0\right)$. Moreover, the (reduced) hydrostatic pressure $p^{*}=1+\phi \chi(1+\alpha)+2 \sqrt{2 / \pi} \phi \chi \Delta_{s}^{*}$. Due to the inclusion of the term $\Delta_{s}^{*} \partial_{\Delta^{*}} \zeta_{0}^{*}$ in Eq. (1), Fig. 4 of the paper should be replaced by the figure below. We observe first that the (scaled) thermal conductivity $\kappa^{*}(\alpha) / \kappa^{*}(1)$ exhibits a nonmonotonic dependency on the coefficient of restitution in contrast to what observed in Fig. 4 of our paper where the ratio $\kappa^{*}(\alpha) / \kappa^{*}(1)$ decreases with increasing inelasticity. In addition, it is also apparent that the influence of density on the (scaled) thermal conductivity of the revised Fig. 4 is slightly larger than the one found in Fig. 4 of our paper.

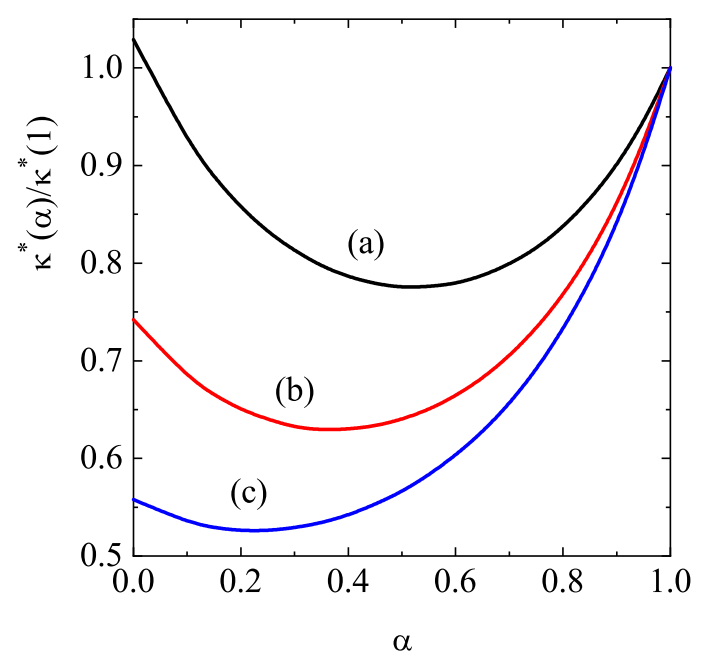

FIG. 4. Plot of the (scaled) thermal conductivity coefficient $\kappa^{*}(\alpha) / \kappa^{*}(1)$ as a function of the coefficient of restitution for three values of the solid volume fraction $\phi$ : (a) $\phi=0$, (b) $\phi=0.2$, and (c) $\phi=0.4$. 